This item was submitted to Loughborough's Research Repository by the author.

Items in Figshare are protected by copyright, with all rights reserved, unless otherwise indicated.

\title{
Motion detection using periodic background estimation subtraction method
}

PLEASE CITE THE PUBLISHED VERSION

https://doi.org/10.1109/CITA.2011.5999523

PUBLISHER

(c) IEEE

VERSION

AM (Accepted Manuscript)

LICENCE

CC BY-NC-ND 4.0

REPOSITORY RECORD

Shazali, Syed Tarmizi Syed, Wai Loon Cheong, Shahrol Mohamaddan, A.M.N. Abg Kamaruddin, A. Yassin, and Keith Case. 2019. "Motion Detection Using Periodic Background Estimation Subtraction Method". figshare. https://hdl.handle.net/2134/32059. 


\section{Motion Detection Using Periodic Background Estimation Subtraction Method}

\author{
S. T. Syed Shazali, W. L. Cheong, S. \\ Mohammaddan, A. M. N. Abg Kamaruddin \\ Department of Mechanical \& Manufacturing Engineering \\ Universiti Malaysia Sarawak \\ Kota Samarahan, Malaysia \\ starmizi@feng.unimas.my,wellancwl86@hotmail.com
}

\author{
K. Case \\ Wolfson School of Mechanical \& Manufacturing \\ Engineering \\ Loughborough University \\ Leicestershire LE11 3TU, United Kingdom \\ k.case@lboro.ac.uk
}

\begin{abstract}
This paper proposed a motion detection system using periodic background estimation subtraction method for outdoor illumination condition using MATLAB. The proposed method is robust to illumination change effect, change in background and noise. The method basically used background subtraction. The background image is estimated at every 0.8 second when the sum of absolute different (SAD) is less than the motion threshold. The input image is luminance normalize before background subtraction. The results were converted into binary image by autothreshold and enhanced the results with dilation and erosion. Blobs were created for each motion objects. Experiment results of using background image estimated by periodic background estimation demonstrate their robustness and effectiveness in background subtraction for real world scene.
\end{abstract}

Keywords- motion detection; periodic background estimation; sum of absolute different; motion threshold; luminance normalize

\section{INTRODUCTION}

There are three commonly used methods to segment the motion area from sequence of image which are the background subtraction, frame differencing, and optical flow segmentation. The most useful, effective and simplest method for detecting moving objects in video images is using background subtraction by comparing the intensity of the background and observed images. However, its application is limited to the background scene that must remain motionless and unchanged [1]. In the real world especially the outdoors is impractical to implement the said methods due to the unpredictable intensity change as illumination fluctuates with the sunlight and weather, tree leaves waving and moving objects becoming static, for example like a moving car parking at a place and stop moving.

Background subtraction method that robustly handles various changes in the background have been proposed by [2][3][4][5]. Frame differencing method were used by [6][7] to detect moving objects. [8] used optical flow segmentation to detect human for safe control of robot. [9] proposed a method to extract moving objects based on exclusive block matching but the tracking is limited because colours of blocks that are the same or similar cannot be avoid.
[1] used an approach by correlation measurement between two blocks of images against varying illuminations with Spatial Modulated Normalized Vector Distance (SMNVD) concept for a background subtraction method in a nonstationary scene. This method is able to detect the moving object but some of the moving regions remained undetected. [3] proposed a method using background subtraction where the background estimated from global illumination change in the observed image by mapping table between the present image and the original background taken in advance. Each pixel from the background subtraction is calculated with mean and variance for each block to get better segmentation but there are errors in detection of background after a moving object passes through and shadow produced by moving object.

[4] used a method for detecting moving objects by using background subtraction that can be applied to cases in which the image has variations due to varying illumination based on two object detection methods which compares the background image and the observed image using invariant features of illumination. It estimates the illumination conditions of the observed image and normalizes the brightness before carrying out background subtraction. [10] developed an algorithm based on constant characteristics of a common, 2-frame interlaced video signal. The proposed algorithm is robust to changes in the lighting conditions, environment and discontinuous motion parameters but large object out of the usual range of interest were segmented into several moving object and the identity of an object is lost when it stopped moving.

In this research, a motion detection method using periodic background estimation subtraction is proposed. It is robust to noise and illumination change effecting the observed image. Objects which are not moving continuously can become background, for example, like a moving car parking at a place and stop moving. The proposed approach is basically using background subtraction where the background image is estimated periodically every 0.8 second when no or less motion using Video and Image Processing Blockset in MATLAB. The background image is estimated using median filter and then, passes through luminance normalizing with the 
observed image before the image is segmented using background subtraction. Then the segmented images were processed using dilation and erosion to remove noise and connect the region of the moving object. The region is further processed as blob to create bounding box and provide centroid coordinate data of each blobs. The system is also able to calculate the total blob in the image.

\section{PERIODIC BACKGROUND ESTIMATION SUBtRACTION METHOD}

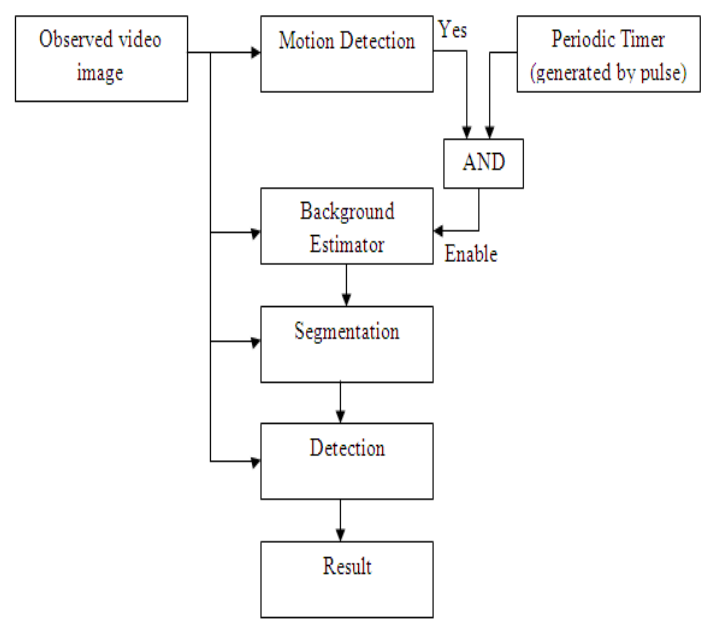

Figure 1. Motion Detection Using Periodic Background Estimation Subtraction Method.

The flow chart of the proposed approach is shown in Figure 1. The first process will be motion detection of the observed image. If the sum of absolute difference of the observed image is more than the motion threshold, the background estimation process will be able to process the background image every 0.8 second generated by the timer. The observed image is segmented with the background subtraction and autothreshold into binary image. The segmented image is further processed in detection to create blob and bounding box to the observed motion object.

\section{A. Motion Detection}

In motion detection, the observed image will be processed by computing 2-D sum of absolute difference (SAD) by shifting image template in single-pixel increments throughout the interior of observed image. The image template is the delayed frame image of the observed image. If the value of $\mathrm{SAD}$ is lower than the motion threshold $\left(17 \times 10^{4} / 255\right)$ with every 0.8 second will enable the background estimator. The pulse generated is a pulse with one amplitude, period of 20 and pulse width of five (5 frames samples). The pulse is shown in Figure 2.

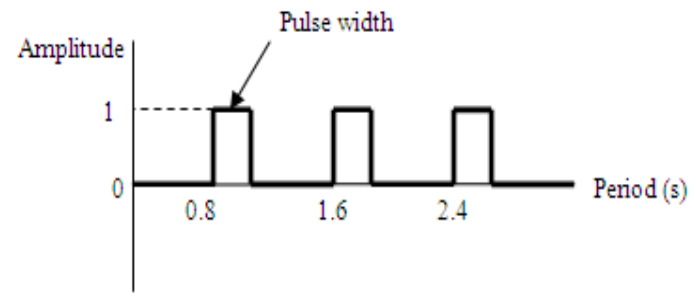

Figure 2. Generated Pulse used as Periodic Timer.

\section{B. Background Estimation}

When the background estimator is initiated, the pulse will take five frames from the observed video images. Each pixel at the same coordinate from the five frames will be used to find the median pixel to produce the background image. The steps are shown in Figure 3.

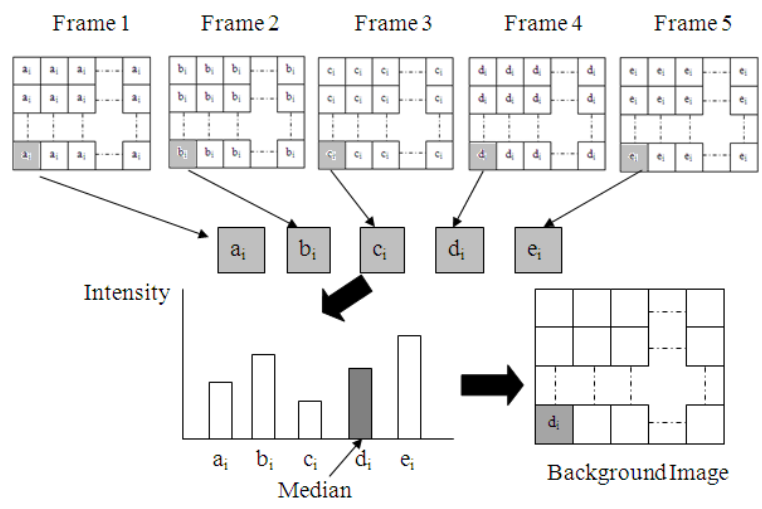

Figure 3. Background Estimation using median.

\section{Segmentation}

In segmentation, the input image will be luminance normalization. The input image is subtracted to find little changes of pixels. These pixels will normalize as background pixels to produce normalized input image. Then, the background subtraction method will be used to segment the motion from the background. The results of the background subtraction are processed by autothreshold to produce binary image from intensity image. Autothreshold used Otsu's method, which determines the threshold by splitting the histogram of the input image to minimize the variance for each of the pixel groups. The binary image is further processed using dilation and erosion to reduce noise in the image and connect the region of moving objects. The results of background subtraction, autothreshold, and dilation and erosion results are displayed in Figure 4. 


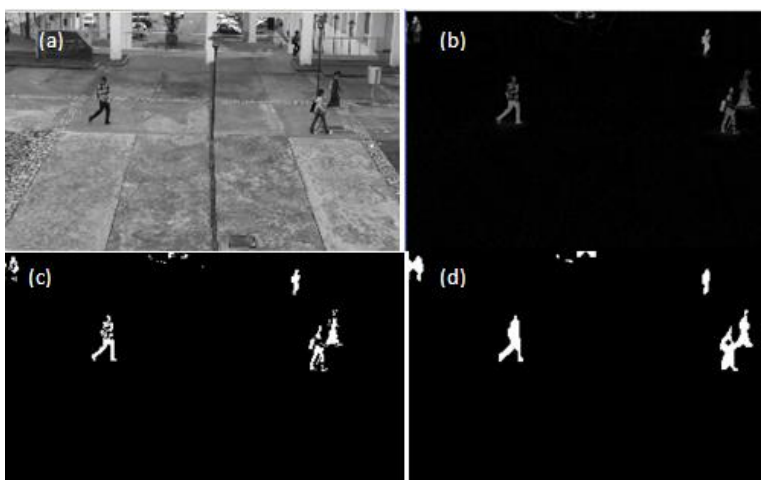

Figure 4. (a) Input video image (b) Background subtraction (c) Autothreshold (d) Dilation and Erosion.

\section{Detection}

In detection, first the object pixels that are close to each other will be merged to create a blob. The minimum blob area in pixels must be 100 pixels. If the pixels are less than 100 pixels, they are considered as noise. The blob which is touching the border of the image is excluded in the analysis. Then the blob is analyzed by calculating the bounding box and centroid. The bounding box of the motion detected is drawn in the output images for each of the blob. Figure 5 and Figure 6 shows the blobs drawn in the binary image and output. Blob counting systems are also included to count the total blob in the image.

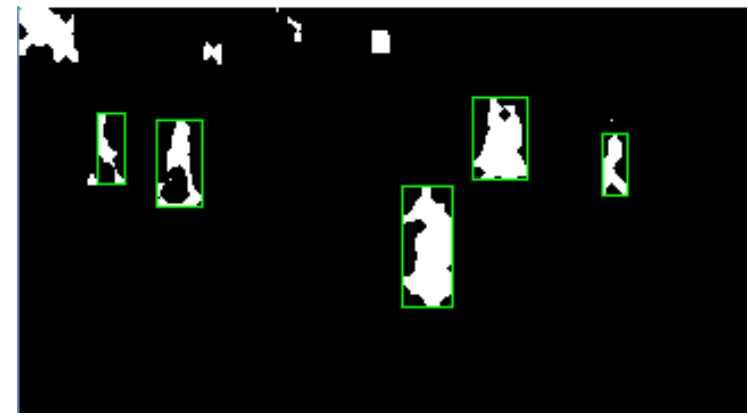

Figure 5. Blobs drawn in the binary image.

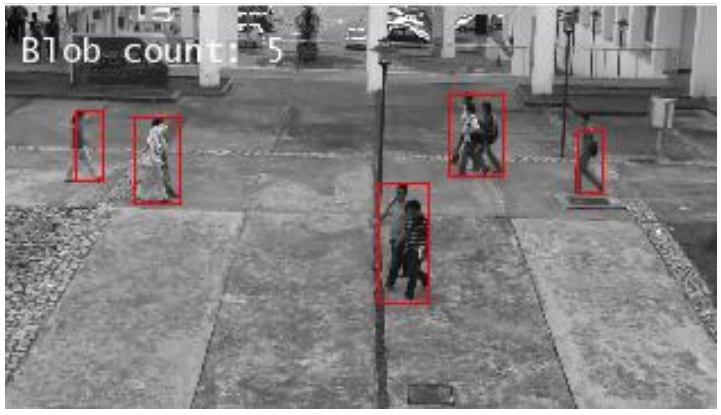

Figure 6. Blobs drawn in the output image.

\section{RESULTS}

The experiment is carried out at Universiti Malaysia Sarawak. A general purpose digital video camera is used as a video recording with $1440 \times 1080$ pixels, 25frames/sec. The resolution of the video image was reduced to $320 \times 180$ pixels for faster processing. The RGB images were processed into gray-scale images. The video images were taken at outdoor illumination condition where the intensity of the light source is the sun. The intensity of the background always changes due to the changing weather conditions. The system is able to count the total of blob in the output image.
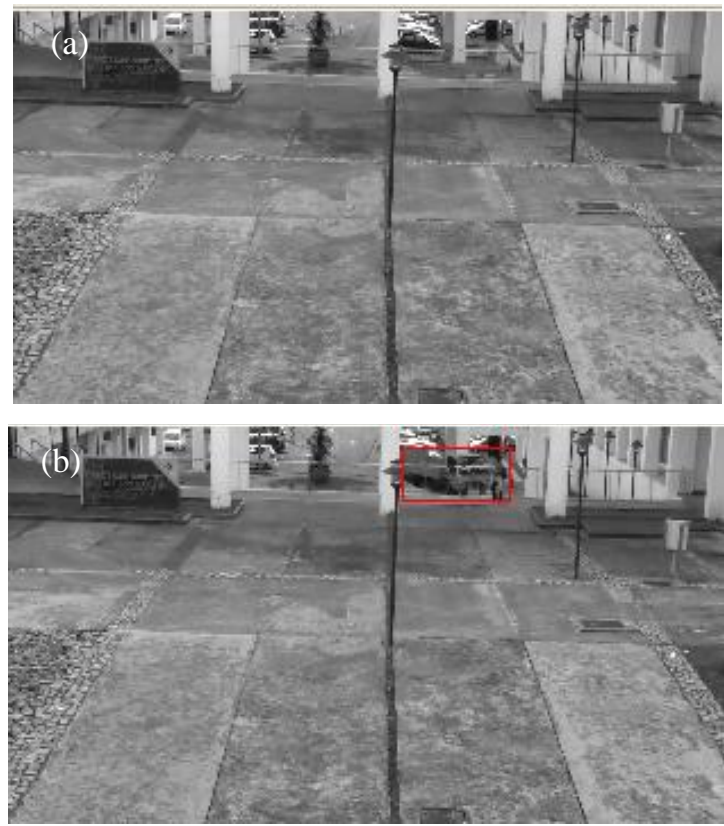

Figure 7. Moving lorry at 8:47:53AM. (a) Background image (b) Tracked image.
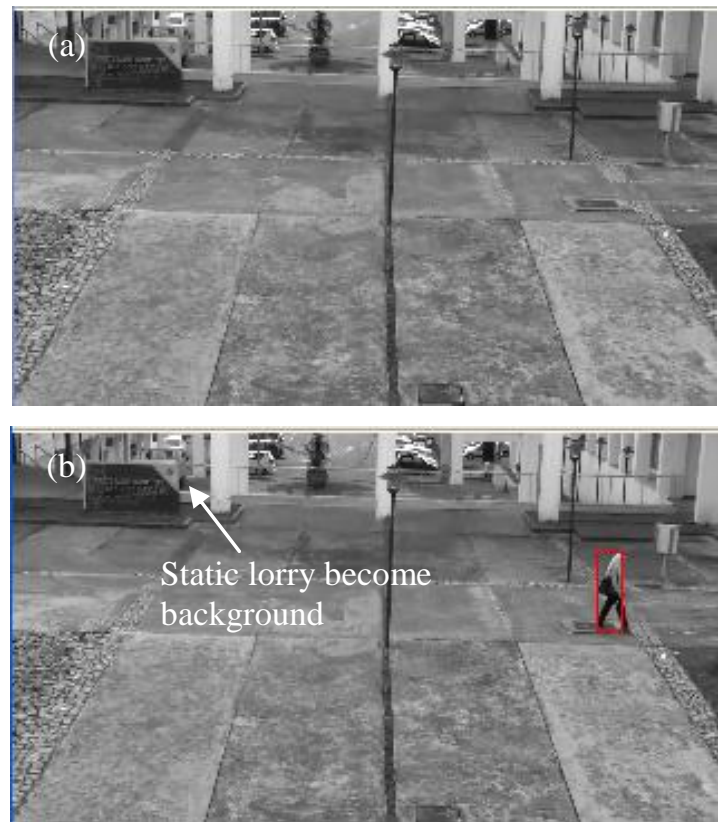

Figure 8 . Moving lorry at 8:48:20AM. (a) Background image (b) Tracked image.

The system is able to detect a moving object at outdoor condition. Object which is in motion that suddenly stops and becomes static eventually becomes the background image. This can be seen in Figure 7 and Figure 8, where the lorry was moving and stop at a place. The background images were able to be estimated at every 0.8 second. The problem with this system is the pedestrian in a static condition are also considered 
as background image until they move. Pedestrian who moved in a group are considered as one blob in the system.

\section{CONCLUSION}

This research paper proposed a method for detecting moving object using periodic background estimation subtraction method. The approach used the background estimation to extract the background image using median filtering periodically every 0.8 second when the sum of absolute difference (SAD) is lower than the motion threshold. The input image is luminance normalize to reduce noise due to illumination. Then, the segmentation is able to segment moving objects using background subtraction and convert to binary image using autothreshold. The binary image is further processed with dilation and erosion for a better result. The result of the object pixels is merged to create a blob for each moving objects.

It have advantages to segment the moving objects under outdoor illumination condition which is always changed and updated the background images every 0.8 second to reduce the noise and objects which is static. When the object stopped moving, it will be unable to detect the object and considered it as background. For future improvement of the system, it should include an improvement in the detection of moving objects and provides an identity for each blob.

\section{ACKNOWLEDGMENT}

This research was supported by Universiti Malaysia Sarawak (UNIMAS) through providing good facilities in conducting the research.

\section{REFERENCES}

[1] T. Matsuyama, T. Ohya, H. Habe, "Background Subtraction for NonStationary Scenes," Kyoto, Japan, 2000.

[2] M. Seki, H. Fujiwara, K. Sumi, "A Robust Background Subtraction Method for Changing Background," IEEE Japan, 2000.

[3] T. Ishikawa, S. Fukui, "Extraction of Moving Objects from Video Sequence Using Estimated Background Brightness," IAPR Workshop on Machine Vision Applications, Nara- ken New Public Hall, Nara, Japan, Dec. 11 - 13, 2002.

[4] T. Matsuyama, T. Wada, H. Habe, K. Tanahashi, "Background Subtraction under Varying Illumination," Systems and Computers in Japan, Vol. 37, No. 4, 2006.

[5] M. Tsuchikawa, A. Sato, H. Koike, A. Tomono, "A Moving-Object Extraction Method Robust Against Illumination Level Changes for a Pedestrian Counting System,” IEEE Japan, 1995.

[6] K. Gupta, A. V. Kulkarni, "Implementation of an Automated Single Camera Object Tracking System Using Frame Differencing and Dynamic Template Matching," India, 2008.

[7] D. J. Shah, D. Estrin, "Motion based bird sensing using frame differencing and Gaussian Mixture," University of California, Riverside : UCR Undergraduate Research Journal, 2008.

[8] M. Takaki, Y. Ohgi, Y. Takahashi, "Vision Based Human Detection for the Safe Control of Robots," Science Links Japan Vol.23 ${ }^{\text {rd }}$, No Page.1B16, Japan, 2005.

[9] Z. Li, K. Yabuta, H. Kitazawa, "A New Method for Moving Object Extraction and Tracking Based on the Exclusive Block Matching," Springer-Verlag Berlin Heidelberg, PSIVT 2009, LNCS 5414, pp. 249260, 2009.

[10] M. Latzel, E. Darcourt, J. K. Tsotsos, "People Tracking using Robust Motion Detection and Estimation," Proceedings of the Second Canadian Conference on Computer and Robot Vision (CRV'05), IEEE, 2005. 